\title{
End-tidal carbon dioxide levels during resuscitation and carbon dioxide levels in the immediate neonatal period and intraventricular haemorrhage
}

\author{
Kentaro Tamura ${ }^{1,2} \cdot$ Emma E Williams $^{1} \cdot$ Theodore Dassios $^{1,3} \cdot$ Anoop Pahuja $^{1} \cdot$ Katie A Hunt $^{1} \cdot$ Vadivelam Murthy $^{1}$.

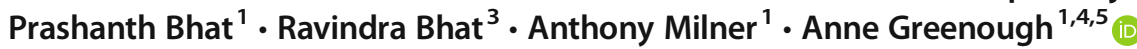

Received: 13 September 2019 / Revised: 29 November 2019 / Accepted: 3 December 2019 / Published online: 17 December 2019

(C) The Author(s) 2019

\begin{abstract}
Abnormal levels of end-tidal carbon dioxide $\left(\mathrm{EtCO}_{2}\right)$ during resuscitation in the delivery suite are associated with intraventricular haemorrhage (IVH) development. Our aim was to determine whether carbon dioxide $\left(\mathrm{CO}_{2}\right)$ levels in the first 3 days after birth reflected abnormal $\mathrm{EtCO}_{2}$ levels in the delivery suite, and hence, a prolonged rather than an early insult resulted in IVH. In addition, we determined if greater $\mathrm{EtCO}_{2}$ level fluctuations during resuscitation occurred in infants who developed IVH. EtCO levels during delivery suite resuscitation and $\mathrm{CO}_{2}$ levels on the neonatal unit were evaluated in 58 infants (median gestational age 27.3 weeks). Delta $\mathrm{EtCO}_{2}$ was the difference between the highest and lowest level of $\mathrm{EtCO}_{2}$. Thirteen infants developed a grade 3-4 IVH (severe group). There were no significant differences in $\mathrm{CO}_{2}$ levels between those who did and did not develop an IVH (or severe IVH) on the NICU. The delta $\mathrm{EtCO}_{2}$ during resuscitation differed between infants with any IVH (6.2 (5.4-7.5) $\mathrm{kPa}$ ) or no IVH $(3.8(2.7-4.3) \mathrm{kPA})(p<0.001)$ after adjusting for differences in gestational age. Delta $\mathrm{EtCO}_{2}$ levels gave an area under the ROC curve of 0.940 for prediction of IVH.

Conclusion: The results emphasize the importance of monitoring $\mathrm{EtCO}_{2}$ levels in the delivery suite.
\end{abstract}

Kentaro Tamura and Emma E. Williams are joint first authors

Clinical Practice: Swallowing problems in cerebral palsy

Communicated by Patrick Van Reempts

\author{
Anne Greenough \\ anne.greenough@kcl.ac.uk \\ Kentaro Tamura \\ tamura@med.u-toyama.ac.jp \\ Emma E Williams \\ emma.e.williams@kcl.ac.uk \\ Theodore Dassios \\ theodore.dassios@kcl.ac.uk \\ Anoop Pahuja \\ anoop.pahuja@nhs.net \\ Katie A Hunt \\ katie.a.hunt@kcl.ac.uk \\ Vadivelam Murthy \\ v.murthy@nhs.net \\ Prashanth Bhat \\ prashanth.bhat@bsuh.nhs.uk
}

Ravindra Bhat

ravindra.bhat@nhs.net

Anthony Milner

anthony.milner@kcl.ac.uk

1 Women and Children's Health, School of Life Course Sciences, Faculty of Life Sciences and Medicine, King's College London, London, UK

2 Division of Neonatology, Maternal and Perinatal Centre, Toyama University Hospital, Toyama, Japan

3 Neonatal Intensive Care Centre, King's College Hospital NHS Foundation Trust, London, UK

4 The Asthma UK Centre in Allergic Mechanisms of Asthma, Kings College London, London, UK

5 NIHR Biomedical Research Centre at Guy's and St Thomas' NHS Foundation Trust and King's College, London, UK 
What is Known:

- Abnormal levels of carbon dioxide $\left(\mathrm{CO}_{2}\right)$ in the first few days after birth and abnormal end-tidal $\mathrm{CO}_{2}$ levels (EtCO $)_{2}$ levels during resuscitation are associated in preterm infants with the risk of developing intraventricular haemorrhage (IVH).

What is New:

- There were no significant differences in NICU $\mathrm{CO}_{2}$ levels between those who developed an IVH or no IVH.

- There was a poor correlation between delivery suite $\mathrm{ETCO}_{2}$ levels and $\mathrm{NICU} \mathrm{CO}_{2}$ levels.

- Large fluctuations in EtCO ${ }_{2}$ during resuscitation in the delivery suite were highly predictive of IVH development in preterm infants.

Keywords Carbon dioxide $\cdot$ End-tidal carbon dioxide $\cdot$ Intraventricular haemorrhage $\cdot$ Prematurity $\cdot$ Resuscitation

$\begin{array}{ll}\text { Abbreviations } \\ \mathrm{CO}_{2} & \text { Carbon dioxide } \\ \mathrm{EtCO}_{2} & \text { End-tidal carbon dioxide } \\ \mathrm{FiO}_{2} & \text { Inspired oxygen fraction } \\ \mathrm{IQR} & \text { Interquartile range } \\ \mathrm{IVH} & \text { Intraventricular haemorrhage } \\ \mathrm{pCO} & \text { Partial pressure of carbon dioxide } \\ \mathrm{PEEP} & \text { Positive end expiratory pressure } \\ \mathrm{RFM} & \text { Respiratory function monitoring } \\ \text { Vte } & \text { Expiratory tidal volume }\end{array}$

Introduction

Improvements in neonatal intensive care have resulted in decreased mortality rates of preterm infants. The development of intraventricular haemorrhage (IVH), however, can result in long-term adverse outcomes. Several studies of preterm infants have demonstrated that abnormal levels of carbon dioxide $\left(\mathrm{CO}_{2}\right)$ within the first few days post-delivery are associated with the development of IVH $[1,2]$. We recently reported that preterm infants who developed an IVH had higher endtidal $\mathrm{CO}_{2}\left(\mathrm{EtCO}_{2}\right)$ levels in the delivery suite during resuscitation [3]. It has not, however, been determined whether abnormal $\mathrm{EtCO}_{2}$ levels during resuscitation of preterm infants translate into abnormal $\mathrm{CO}_{2}$ levels over the subsequent $72 \mathrm{~h}$ after birth, and hence, whether it is a prolonged rather than an early insult which results in the development of IVH. Our aim then was to study $\mathrm{EtCO}_{2}$ levels in the delivery suite during resuscitation of preterm infants and their relationship to the subsequent $\mathrm{CO}_{2}$ levels within the first 3 days after birth and the development of IVH.

In one study of very low birth weight infants, not only high but also low partial pressures of carbon dioxide $\left(\mathrm{pCO}_{2}\right)$ were associated with severe IVH development, as was a greater degree of fluctuation in $\mathrm{pCO}_{2}$ levels within the first 4 days after birth [2]. In term infants, undergoing therapeutic hypothermia for hypoxic ischaemic encephalopathy, adverse neurodevelopmental outcomes were also associated with a greater degree of $\mathrm{pCO}_{2}$ variability [4]. Preterm and unstable infants are more susceptible to changes in cerebral blood flow and display pressure passivity [5]. Furthermore, fluctuations of $\mathrm{pCO}_{2}$ can adversely affect cerebral oxygenation and perfusion and the electrical activity of the brain [6]; such fluctuation is predictive of adverse neurodevelopmental outcomes [7]. We have demonstrated that not only high $\mathrm{EtCO}_{2}$ levels during resuscitation in the delivery suite were associated with IVH development but also inflations resulting in larger tidal volumes and lower $\mathrm{EtCO}_{2}$ levels [3]. Those findings were subsequently confirmed by Mian and colleagues who found that tidal volumes in excess of $6 \mathrm{ml} / \mathrm{kg}$ were associated with cerebral damage in the neonatal period [8]. A further aim, therefore, of this study then was to test the hypothesis that infants exposed to the largest fluctuations in $\mathrm{EtCO}_{2}$ levels during resuscitation in the delivery suite would be at greatest risk of developing an IVH.

\section{Methods}

We reviewed the records of infants born at less than or equal to 33 weeks of gestational age who required resuscitation in the delivery suite between March 2010 and March 2014. A certain part of their data was reported in a previous paper [3]. Ethical approval was given by the Outer London Ethics Committee for the data collection. The committee required parental consent only for analysis of the data, which was obtained once the mother was transferred to the postnatal ward. This study was approved by the King's College Hospital NHS Foundation Trust Research and Audit Department.

\section{Resuscitation protocol}

The resuscitation protocol, together with the monitoring methodology, has previously been described [3]. The clinicians attending the resuscitations had all been trained in newborn life support and had received the Resuscitation Council (UK) Newborn Life Support provider certificate. They had also been trained to operate the respiratory function monitor. During the resuscitation, the respiratory function monitor was set to display tidal volume, flow, inflation pressure and $\mathrm{EtCO}_{2}$ levels.

\section{Resuscitation monitoring}

The resuscitation recordings were analysed to determine the duration of resuscitation, the number of inflations, the 
inflation pressure, the positive end expiratory pressure (PEEP), inflation time (Ti), mean airway pressure (MAP), expiratory tidal volume $\left(\mathrm{V}_{\mathrm{TE}}\right)$ of each inflation and the breath-by-breath minute ventilation. The highest $\mathrm{EtCO}_{2}$ was recorded as were the number of inflations with tidal volumes more than $6 \mathrm{mls} / \mathrm{kg}$ (high tidal volumes) or end-tidal $\mathrm{CO}_{2}$ levels of less than $4.5 \mathrm{kPa}$ (low EtCO ${ }_{2}$ levels) and the number of inflations with both high tidal volumes and low $\mathrm{EtCO}_{2}$ levels as previously described [3]. The degree of fluctuation of $\mathrm{EtCO}_{2}$ (delta $\mathrm{EtCO}_{2}$ ) during resuscitation was determined by calculating the difference between the highest and lowest $\mathrm{EtCO}_{2}$ value during resuscitation in delivery suite.

\section{NICU monitoring}

The unit's routine policy was to insert arterial lines for blood gas sampling, and in the absence of such, access capillary sampling was undertaken. Blood gases were recorded on the intensive care observation charts every 4 to $6 \mathrm{~h}$ depending on the clinical status of the infant, and the hourly ventilator settings were collected at the time as each arterial gas result. Venous blood gases were not analysed in this study. Data on the maximum, minimum and average $\mathrm{pCO}_{2}$ value on each of the first, second and third days after birth were collected. The degree of fluctuation of $\mathrm{CO}_{2}$ (delta $\mathrm{CO}_{2}$ ) during each of the first 3 postnatal days was determined by calculating the difference between the highest and lowest $\mathrm{CO}_{2}$ value on each day. Volume targeted ventilation was not used on the NICU during the study period.

The medical records were examined to determine whether the infants developed an IVH which was diagnosed from cranial ultrasounds. As per unit policy, cranial ultrasounds were performed on admission to the unit for all infants born below 34 weeks of gestation. Cranial ultrasounds were repeated on day 3 , between days 7 and 10, days 14 and 21 and days 28 and 30. All cranial ultrasounds were reported by a neonatal consultant. The IVHs were reported as grades I to IV using the Papile classification [9]. Severe IVH was diagnosed as a grade III or IV IVH. Other data extracted from the medical records included the infant's gestational age, birth weight and Apgar score at $5 \mathrm{~min}$, and whether the mother had received corticosteroids antenatally or had chorioamnionitis.

\section{Statistical analysis}

The data were tested for normality using the KolmogorovSmirnov test and found to be non-normally distributed; hence, differences were assessed for statistical significance using the Mann-Whitney $U$ test or the chi-square test as appropriate. As infants who develop IVH tend to be more immature, regression analyses were performed to determine if the results remained statistically significant after correcting for differences in gestational age. The strength of any relationships of
$\mathrm{EtCO}_{2}$ values during resuscitation with $\mathrm{CO}_{2}$ levels during the first 3 days after birth were assessed by calculating Spearman correlation coefficients. The performance of factors identified as significantly related to IVH development from the regression analysis were assessed by receiver operator characteristic curve analysis and estimation of the corresponding area under the curve (AUC). Statistical analyses were performed using JMP statistical software (JMP 13.0.0, SAS Institute Inc., NC, USA) and SPSS software 25.0 (SPSS Inc., Chicago IL, USA).

\section{Results}

Three hundred and seventy infants were born at less than or equal to 33 weeks of gestation within the study period. There were 150 infant recordings available for analysis, but 56 had recordings of tidal volume or $\mathrm{EtCO}_{2}$ traces which were unsuitable for analysis, a further 24 were excluded due to a large leak throughout and 12 excluded due to missing neonatal unit observation charts. The remaining 58 infants were included in this study and had a median gestational age of 27.3 (interquartile range (IQR) 24.9-29.0) weeks and a birth weight of 0.90 $(0.72-1.21) \mathrm{kg}$. The infants who were and were not included in this study did not differ significantly with regard to birth weight $(p=0.893)$ or gestational age $(p=0.785)$. Fifty-three of the 58 infants were ventilated on day 1 after birth. Thirty infants developed no IVH with 28 infants developing an IVH. Seven infants developed a grade 1 , eight infants grade 2 , five infants grade 3 and the remaining eight infants a grade $4 \mathrm{IVH}$, that is 13 infants developed a severe IVH. All infants who developed an IVH did so in the first 3 days after birth. Eleven infants developed an IVH on day 1 (9 grade $1-2)$, nine on day 2 (5 grade $1-2$ ) and eight on day 3 (1 grade $1-2)$. There were no significant differences in $\mathrm{CO}_{2}$ levels on the NICU and IVH development.

The highest $\mathrm{EtCO}_{2}$ during resuscitation in the no-IVH group was $8.0(7.1-8.4) \mathrm{kPa}$ and $10.0(8.9-11.2) \mathrm{kPa}$ in the IVH group; the difference remained statistically significant after adjusting for differences in gestational age between the two groups $(p=0.0002)$. The highest $\mathrm{EtCO}_{2}$ during resuscitation in the no/non-severe group IVH was $8.2(7.4-9.9) \mathrm{kPa}$ and $10.2(8.6-11.1) \mathrm{kPa}$ in the severe IVH group; the difference remained statistically significant after adjusting for differences in gestational age between the two groups ( $p=$ 0.037). There was no significant difference in the mean tidal volume during resuscitation between infants in the no/non severe IVH group and the severe IVH group ( $p=0.621)$.

The lowest $\mathrm{EtCO}_{2}$ during resuscitation in the no-IVH group was $4.2(3.2-5.2) \mathrm{kPa}$ and $3.5(3.0-4.5) \mathrm{kPa}$ in the IVH group, but this was not statistically significant after adjusting for the difference in gestational age between the two groups $(p=0.11)$. The lowest $\mathrm{EtCO}_{2}$ during resuscitation in the no/non-severe IVH group was $4.0(3.2-5.1) \mathrm{kPa}$ and 3.1 
$(2.7-3.5) \mathrm{kPa}$ in the severe IVH group; the difference remained significant after adjusting for the difference in gestational age between the two groups $(p=0.041)$.

There were weak correlations between the highest end-tidal $\mathrm{CO}_{2}$ on delivery suite and the highest $\mathrm{pCO}_{2}$ on the neonatal unit on days $2(r=0.274, p=0.047)$ and $3(r=0.383, p=$ 0.005).

The delta $\mathrm{EtCO}_{2}$ during resuscitation was significantly different between the no/non-severe IVH $(4.3(3.1-5.6) \mathrm{kPa})$ and severe IVH groups $(7.2(5.9-7.6) \mathrm{kPa})(p=0.003)$ and between infants with any IVH (6.2 (5.4-7.5) kPa) or no IVH (3.8 $(2.7-4.3) \mathrm{kPa})(p<0.001)$ after adjusting for differences in gestational age. The delta $\mathrm{CO}_{2}$ level during resuscitation weakly correlated with the delta $\mathrm{CO}_{2}$ level on the neonatal unit on days $2(r=0.385, p=0.004)$ and $3(r=0.423, p=$ 0.002 ).

The receiver operator characteristic curve analysis demonstrated that in predicting any IVH, the maximum level of $\mathrm{EtCO}_{2}$ on the delivery suite had an AUC of 0.856 and the delta $\mathrm{CO}_{2}$ during resuscitation had an AUC of 0.940. A delta $\mathrm{EtCO}_{2}$ equal or greater than $4.9 \mathrm{kPa}$ predicted any IVH development with $96.4 \%$ sensitivity and $83.3 \%$ specificity.

\section{Discussion}

We have demonstrated that preterm infants who developed an IVH had a higher maximum level of $\mathrm{EtCO}_{2}$ and greater degree of fluctuation of $\mathrm{EtCO}_{2}$ levels during resuscitation. In addition, we highlight that the delta $\mathrm{EtCO}_{2}$ level in the delivery suite had both a high sensitivity and specificity in predicting IVH development. Importantly, we have shown that abnormal $\mathrm{EtCO}_{2}$ levels during resuscitation did not necessarily translate into abnormal levels on the neonatal unit. Our findings, then suggest that it is early abnormal carbon dioxide levels in preterm infants that are important in IVH development. Previously, initial post-delivery levels of $\mathrm{pCO}_{2}$ were found to be significantly higher in infants with cerebral haemorrhage than those without, but by $8 \mathrm{~h}$ after birth, those differences were no longer significant [10], which are consistent with our findings of the significant association of abnormal $\mathrm{CO}_{2}$ levels in the delivery suite, but not the neonatal unit, with IVH development. Some studies have suggested that sicker infants undergo more blood gas analysis than more stable infants, hence, allowing detection of a greater degree of fluctuation in levels of carbon dioxide [2]. We continuously monitored $\mathrm{EtCO}_{2}$ during resuscitation in all infants included in our cohort, and therefore, the outcomes relating to fluctuation of carbon dioxide are not influenced by the infant's degree of sickness. We speculate that by the time infants are admitted to the neonatal unit, clinicians have greater access to routine monitoring and would readily re-check abnormal levels of $\mathrm{pCO}_{2}$ by blood gas analysis, hence, limiting the degree of fluctuation between extremes of values and thereby achieving tighter control. This would explain our findings that it is the delivery suite levels of $\mathrm{CO}_{2}$ that are more influential regarding IVH development and why the extreme levels of carbon dioxide seen during resuscitation do not translate to neonatal unit levels of $\mathrm{CO}_{2}$ during the subsequent $72 \mathrm{~h}$. We did not use volume targeted ventilation during the study period and this would have likely reduced fluctuations in $\mathrm{CO}_{2}$ levels on the NICU.

As previously mentioned [3], we did find low $\mathrm{EtCO}_{2}$ levels that were associated with IVH development, but this was no longer significant after adjustment for gestational age. Nevertheless, we highlight fluctuations in $\mathrm{EtCO}_{2}$ that are highly predictive of IVH development. Hence, it is important to avoid both high and low levels of $\mathrm{EtCO}_{2}$ during resuscitation. Our results emphasize that respiratory function monitoring should be helpful in the labour suite. It has been previously shown that resuscitators were unable to accurately assess chest wall movement $[11,12]$, and use of respiratory function monitoring (RFM) can result in less mask leak and a lower rate of excessive tidal volumes [13]. We have, however, previously emphasized that resuscitators must have appropriate training so they can accurately interpret the results of such monitoring in real time [14]. Eye tracking technology has demonstrated that providers spend more time looking at the exhaled tidal volume waveform data than any other RFM parameter to guide positive pressure ventilation [15] suggesting further training is required on the importance of $\mathrm{EtCO}_{2}$ levels during resuscitation.

Our study has strengths and some limitations. This is the first study to evaluate $\mathrm{EtCO}_{2}$ levels and fluctuation of $\mathrm{EtCO}_{2}$ levels during resuscitation and how they correlated with both carbon dioxide levels in the first 3 days after birth and IVH development. We monitored $\mathrm{EtCO}_{2}$ levels during resuscitation, yet on the neonatal unit, we have analysed levels of $\mathrm{CO}_{2}$ from blood gas measurements. Previous studies, however, have found $\mathrm{EtCO}_{2}$ to be as accurate as arterial monitoring when trending carbon dioxide levels [16]. We did not, however, measure cerebral blood flow in our cohort and hence, cannot speculate on the mechanisms behind our findings.

In conclusion, a greater degree of fluctuation of $\mathrm{EtCO}_{2}$ levels in the delivery suite was associated with a significantly increased risk in the development of IVH. Those results emphasise the need for $\mathrm{EtCO}_{2}$ monitoring during resuscitation in the delivery suite.

\section{Authors' contributions KT analysed the data}

EW analysed the data

VM collected the data

PB collected the data

$\mathrm{RB}$ assisted in collection of the data

TD assisted in analysis of the data

AG designed the study and analysed the data

ADM designed the study and analysed the data

All authors were involved in writing the manuscript and approved the final version 
Funding information Dr Murthy was supported by a grant from the Guy's and St Thomas' Charity. Dr Bhat, Dr Hunt and Dr Williams were supported by grants from the Charles Wolfson Charitable Trust. Additionally, Drs Hunt, Williams and Tamura were supported by SLE. Dr Tamura held an SLE SATA fellowship. The research was supported by the National Institute for Health Research (NIHR) Biomedical Research Centre based at Guy's and St Thomas' NHS Foundation Trust and King's College London. The views expressed are those of the author(s) and not necessarily those of the NHS, the NIHR or the Department of Health.

\section{Compliance with ethical standards}

Conflict of interest The authors declare that they have no conflicts of interest.

Ethical approval All procedures performed in studies involving human participants were in accordance with the ethical standards of the institutional and/or national research committee and with the 1964 Helsinki declaration and its later amendments or comparable ethical standards.

Statement of informed consent Parents gave informed written consent.

Open Access This article is licensed under a Creative Commons Attribution 4.0 International License, which permits use, sharing, adaptation, distribution and reproduction in any medium or format, as long as you give appropriate credit to the original author(s) and the source, provide a link to the Creative Commons licence, and indicate if changes were made. The images or other third party material in this article are included in the article's Creative Commons licence, unless indicated otherwise in a credit line to the material. If material is not included in the article's Creative Commons licence and your intended use is not permitted by statutory regulation or exceeds the permitted use, you will need to obtain permission directly from the copyright holder. To view a copy of this licence, visit http://creativecommons.org/licenses/by/4.0/.

\section{References}

1. Erickson SJ, Grauaug A, Gurrin L, Swaminathan M (2002) Hypocarbia in the ventilated preterm infant and its effect on intraventricular haemorrhage and bronchopulmonary dysplasia. $\mathrm{J}$ Paediatr Child Health 38:560-562

2. Fabres J, Carlo WA, Phillips V, Howard G, Ambalavanan N (2007) Both extremes of arterial carbon dioxide pressure and the magnitude of fluctuations in arterial carbon dioxide pressure are associated with severe intraventricular hemorrhage in preterm infants. Pediatrics 119:299-305

3. Pahuja A, Hunt K, Murthy V, Bhat P, Bhat R, Milner AD, Greenough A (2018) Relationship of resuscitation, respiratory function monitoring data and outcomes in preterm infants. Eur $\mathrm{J}$ Pediatr 177:1617-1624
4. Hansen G, Al Shafouri N, Narvey M, Vallance JK, Srinivasan G (2016) High blood carbon dioxide variability and adverse outcomes in neonatal hypoxic ischemic encephalopathy. J Matern Fetal Neonatal Med 29:680-683

5. Tsuji M, Saul JP, du Plessis A, Eichenwald E, Sobh J, Crocker R, Volpe JJ (2000) Cerebral intravascular oxygenation correlates with mean arterial pressure in critically ill premature infants. Pediatrics 106:625-632

6. Dix LML, Weeke LC, de Vries LS, Groenendaal F, Baerts W, van Bel F, Lemmers PMA (2017) Carbon dioxide fluctuations are associated with changes in cerebral oxygenation and electrical activity in infants born preterm. J Pediatr 187:66-72

7. McKee LA, Fabres J, Howard G, Peralta-Carcelen M, Carlo WA, Ambalavanan N (2009) $\mathrm{PaCO}_{2}$ and neurodevelopment in extremely low birth weight infants. J Pediatr 155:217-221

8. Mian Q, Cheung PY, O'Reilly M, Barton SK, Polglase GR, Schmolzer GM (2019) Impact of delivered tidal volume on the occurrence of intraventricular haemorrhage in preterm infants during positive pressure ventilation in the delivery room. Arch Dis Child Fetal Neonatal Ed 104:F57-F62

9. Papile LA, Burstein J, Burstein R, Koffler H (1978) Incidence and evolution of subependymal and intraventricular hemorrhage: a study of infants with birth weights less than 1500 gm. J Pediatr 92:529-534

10. Van de Bor M, Van Bel F, Lineman R, Ruys JH (1986) Perinatal factors and periventricular-intraventricular hemorrhage in preterm infants. Am J Dis Child 140:1125-1130

11. Poulton DA, Schmolzer GM, Morley CJ, Davis PG (2011) Assessment of chest rise during mask ventilation of preterm infants in the delivery room. Resuscitation 82:175-179

12. Schmölzer GM, Kamlin OC, O’Donnell CP, Dawson JA, Morley CJ, Davis PG (2010) Assessment of tidal volume and gas leak during mask ventilation of preterm infants in the delivery room. Arch Dis Child Fetal Neonatal Ed 95:F393-F397

13. Schmolzer GM, Morley CJ, Wong C, Dawson JA, Kamlin CO, Donath SM, Hooper SB, Davis PG (2012) Respiratory function monitor guidance of mask ventilation in the delivery room: a feasibility study. J Pediatr 160:377-381

14. Milner A, Murthy V, Bhat P, Fox G, Campbell ME, Milner AD, Greenough A (2015) Evaluation of respiratory function monitoring at the resuscitation of prematurely born infants. Eur J Pediatr 174: 205-208

15. Katz TA, Weinberg DD, Fishman CE, Nadkarni V, Tremoulet $P, T e$ Pas AB, Sarcevic A, Foglia EE (2019) Visual attention on a respiratory function monitor during simulated neonatal resuscitation: an eye-tracking study. Arch Dis Child Fetal Neonatal Ed 104:F259F264

16. Rozycki HJ, Sysyn GD, Marshall MK, Malloy R, Wiswell TE (1998) Mainstream end-tidal carbon dioxide monitoring in the neonatal intensive care unit. Pediatrics 101:648-653

Publisher's note Springer Nature remains neutral with regard to jurisdictional claims in published maps and institutional affiliations. 